\title{
Electrothermal X-Parameters for Dynamic Modeling of RF and Microwave Power Transistors
}

\author{
Sean J. Gillespie ${ }^{1}$, David E. Root ${ }^{1,2}$, Mihai Marcu ${ }^{2}$, Peter H. Aaen ${ }^{1}$ \\ ${ }^{1}$ Department of Electrical and Electronic Engineering, University of Surrey, Guildford, UK \\ ${ }^{2}$ Keysight Technologies, Inc., Santa Rosa, CA, USA \\ s.gillespie@surrey.ac.uk, david_root@keysight.com, mihai_marcu@keysight.com, p.aaen@surrey.ac.uk
}

\begin{abstract}
For the first time, this paper presents and validates a novel extension of the $X$-parameter behavioral modeling paradigm to include dynamic electro-thermal phenomena, a key source of long-term memory affecting transistors. The dynamic thermal X-parameter model (DTXM) adds a novel but straightforward method to implement envelope domain sub-circuit in a feedback loop around a conventional static X-parameter model, enabling the simulation of modulated waveform-dependent dynamic self-heating effects. The extended model is identified from conventional $\mathrm{CW}$ or pulsed $\mathrm{X}$-parameter measurements, over a range of ambient temperatures. A re-referencing of the extracted $\mathrm{X}$-parameter data to the junction temperature is performed, based on estimated or a calculated thermal resistance and thermal capacitance. The model can also be generated in the simulation environment starting from a dynamic electro-thermal compact time-domain model. The DTXM accounts for thermallyinduced asymmetry of intermodulation distortion products and temperature hysteresis depending on the signal bandwidth.

Keywords - behavioural modelling, X-Parameters, electrothermal device modelling, NVNA.
\end{abstract}

\section{INTRODUCTION}

Accurate and robust large-signal transistor models are essential tools for first-pass design success of advanced microwave and RF circuits, such as power amplifiers, that are based on new power transistor technologies [1]. Useful semiconductor technologies are often available for commercial applications long before robust, physically-based compact transistor models are implemented in simulators for general use by designers. It may take many years to develop accurate compact models for a given technology and still longer to have models and their parameter extraction flows robustly implemented in commercial modelling CAE SW. Every type of device (e.g. Si MOSFET, GaN HEMT, etc.) requires a different compact model. It is a daunting task to generate and implement such a multiplicity of distinct models at the rapid rate the semiconductor technologies are introduced and enhanced.

Behavioural models present an attractive alternative to conventional compact transistor models [2], [3]. Behavioural models apply to a very wide variety of technologies and have been shown to simulate more rapidly and with more robust convergence properties, even when compared to compact models from which they can be faithfully generated. They retain the accuracy of the underlying large-signal measurements (or simulations) from which they are generated. Examples of widely accepted behavioural models suitable for advanced RF and microwave large-signal design of Power Amplifiers (PA) are X-parameters [4] and the Cardiff model [5]. X-parameters in ADS are used in this work, but the approach is general.

The X-parameter behavioural model is defined by a set of a time-invariant nonlinear spectral maps formulated in the frequency-domain [4] and constructed from large-signal measurements on an NVNA [6] or generated from models in the circuit simulator. X-parameters capture the non-linear characteristics of a device-under-test (DUT), including compression, harmonic and intermodulation distortion generation, under static conditions. At present, a frequently cited limitation of the X-parameter paradigm is the lack of commercial implementation of dynamic memory effects, such as those due to trapping and self-heating mechanisms, despite some promising research results in the literature [7], [8].

Thermal dependences of transistor characteristics are of great importance for several reasons. Firstly, electrical properties (e.g. power gain, power added efficiency, intermodulation spectra) all depend on the temperature.

The mutual interaction of modulated electrical characteristics and the time-dependent thermal response must be solved self-consistently to properly account, for example, for the known spectral asymmetry of intermodulation distortion products that can be a consequence of dynamic thermal memory [9]. This is done routinely by specialized time-domain compact transistor models, but behavioural modelling approaches to thermal dynamics have been lacking.

For the first time, we develop a dynamic electrothermal behavioural model, capable of predicting the thermal memory and spectral asymmetry phenomena that is prevalent in power transistors.

\section{Methodology}

\section{A. Enhanced Model for Dynamic Electro-Thermal Effects}

A simplified diagram of the dynamic thermal X-parameter model is shown in Fig. 1. The element labelled "Static_XP_Model" in Fig. 1a is a conventional X-parameter large-signal model with $2 \mathrm{RF}$ ports and a third $\mathrm{DC}$ port. In this case, however, the DC voltage corresponds to a temperature. Moreover, the datafiles read by the X-parameter model have been re-parameterized in terms of a junction temperature, rather 


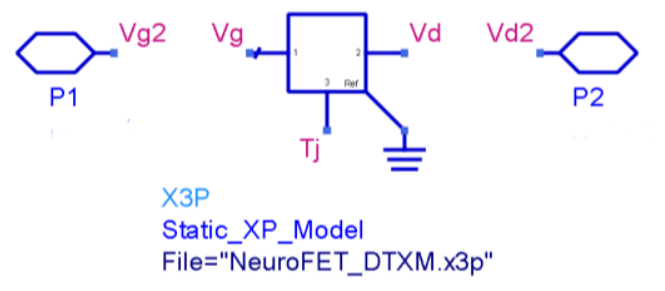

(a) Static X-parameter model
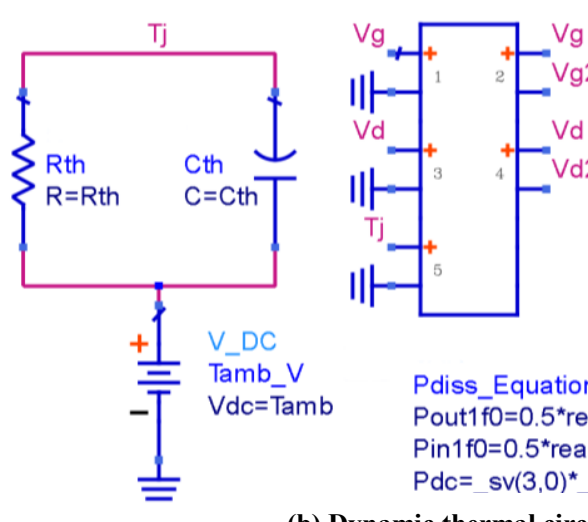

FDD5P

Pdiss_FDD

$\mid[1,0]=0$

$[1,1]=0$

$V[2,0]=\_s v(2,0)$

$\mathrm{V}[2,1]=\_s v(2,1)+$ _sv $(2$, ,harm $)$

$\mid[3,0]=0$

$\mid[3,1]=0$

$\mathrm{V}[4,0]=\_s v(4,0)$

$V[4,1]=\_s v(4,1)+$ sv $(4$, ,harm $)$

$\mid[5,0]=P d c+P i n 1 f 0-P o u t 1 f 0$

Pdiss_Equations

Pout1fO $=0.5^{\star}$ real $\left(\right.$ sv $(3,1)^{\star} \operatorname{conj}(-$ si $\left.(4,1))\right)$

Pin $1 \mathrm{fO}=0.5^{\star}$ real(_sv $\left.(1,1)^{*} \operatorname{conj}(\operatorname{si}(2,1))\right)$

$\mathrm{Pdc}=\_\mathrm{sv}(3,0)^{\star} \_\mathrm{si}(4,0)+\_\mathrm{sv}(1,0)^{\star} \_\mathrm{si}(2,0)$

(b) Dynamic thermal circuit

Fig. 1. ADS circuit model for electrothermal dynamic X-parameter (DTXM) integration. Only two harmonic components are specified for simplicity. The voltages applied to the X-parameter model in (a) are used by the thermal circuit (b) to compute the power dissipation and resulting junction temperature. This is coupled back to the X-parameter model and solved self-consistently during each time step of a circuit envelope simulation.

than the ambient temperature that was controlled in the data acquisition. This portion is described in Section C.

The dynamic thermal circuit implementation, shown in Fig. $1 \mathrm{~b}$, uses a thermal sub-circuit coupled to a novel frequency domain symbolic device (FDD) in ADS that performs a timedependent calculation of the dissipated electrical power in the envelope domain.

\section{B. Thermal X-Parameter Measurements}

Temperature-dependent X-parameter measurements were obtained from a Broadcom ATF-54143 [10] pHEMT power transistor. The measurement setup and key equipment is labelled in Fig. 2. A hot plate was used to perform extraction at discrete ambient temperatures, from $30^{\circ} \mathrm{C}$ to $70^{\circ} \mathrm{C}$, which are used to parameterize the X-parameter file.

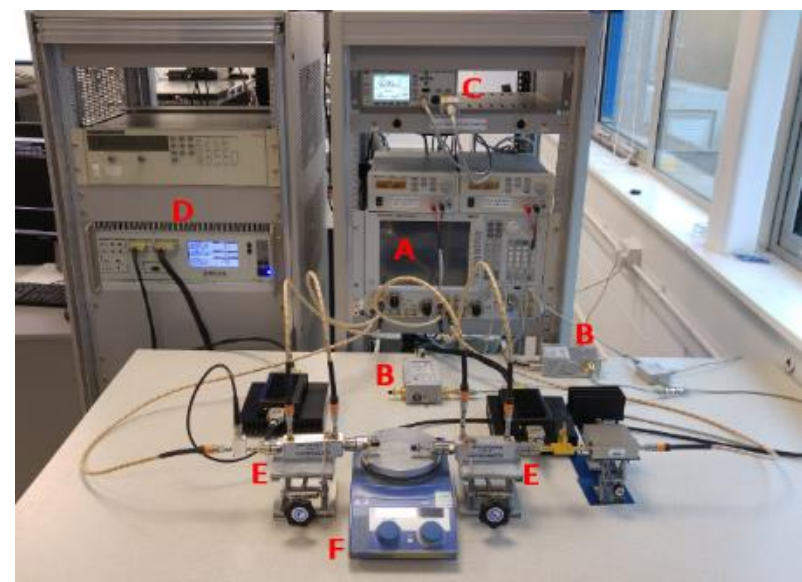

Fig. 2: Equipment used to measure temperature-dependent X-parameters. Labelled to highlight key equipment: A) Keysight N5247a, B) U9391G Comb Generators, C) power meter, D) AMCAD PIV system, E) LLC18-7 couplers, and F) hot plate.

Temperature-dependent data for three different $\mathrm{X}$-parameter terms $\left(X^{\mathrm{F}}, X^{\mathrm{S}}\right.$, and $\left.X^{T}\right)$ are shown in Fig. 3. These terms govern the non-linear input-output relationship of the DUT; where $X^{F} /\left|A_{11}\right|$ relates to the power gain for perfectly matched output impedance, and the $X^{S}$ and $X^{T}$ parameters are sensitivity terms related to mismatch effects [4].
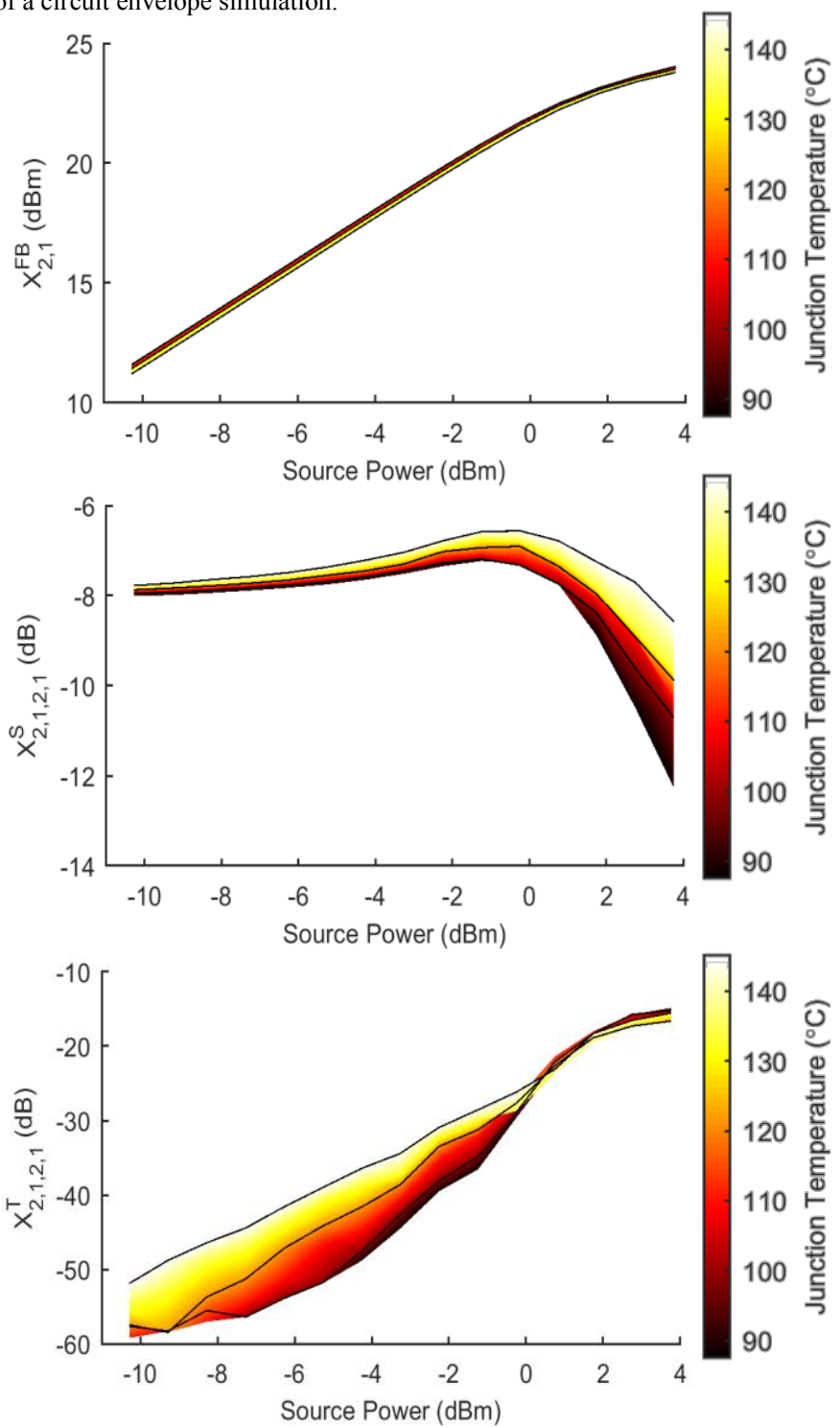

Fig. 3: Measured temperature dependent X-parameters of ATF-54143 power transistor. Showing $\mathrm{X}^{\mathrm{F}}$, related to gain via $\mathrm{X}^{F} /\left|A_{11}\right|$, and the $\mathrm{X}^{\mathrm{S}}$ and $\mathrm{X}^{\mathrm{T}}$ match sensitivity terms of port 2 at the fundamental frequency. 


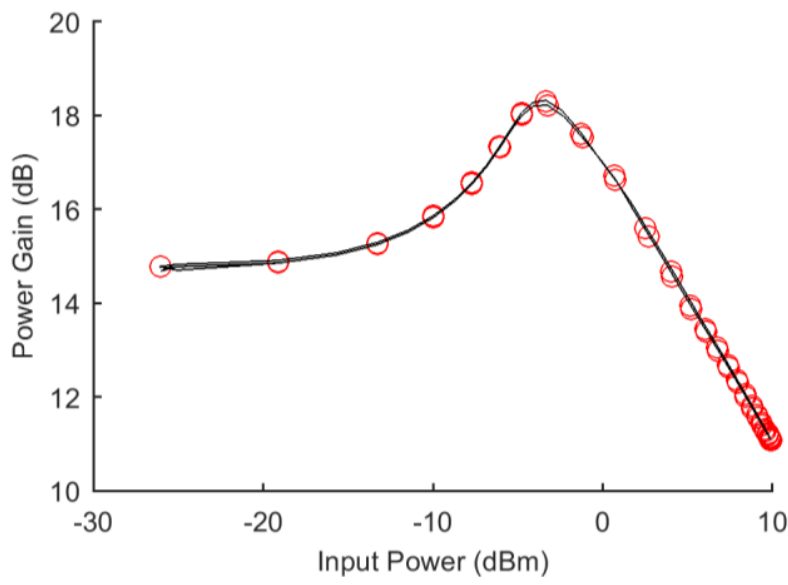

Fig. 4. Dynamic simulation of two-tone response of new dynamic thermal X-parameter model (DTXM) (symbols) and the compact time-domain self-heating model (solid line). The frequency difference between the two tones was $100 \mathrm{kHz}$, corresponding to the thermal time-constant of the device.

\section{Thermal Re-referencing to Junction Temperature}

The DTXM requires the junction temperature to be the independent thermal variable over which the X-parameter values are tabulated, rather than the ambient temperature. This requires nonlinear computations performed as a post-extraction data processing step. Under $\mathrm{CW}$ measurement conditions, for a given (measured or computed) power added efficiency (PAE) and power dissipation, and estimated thermal resistance, $R_{\mathrm{th}}$, this can be computed using the formulas that follow,

$$
\begin{gathered}
P_{\text {diss }}=\left(1-\eta_{\mathrm{PAE}}\right) P_{\mathrm{DC}}=P_{\mathrm{DC}}+P_{\mathrm{in}}-P_{\text {out }} \\
T_{j}=T_{0}+R_{\mathrm{th}} P_{\text {diss }} .
\end{gathered}
$$

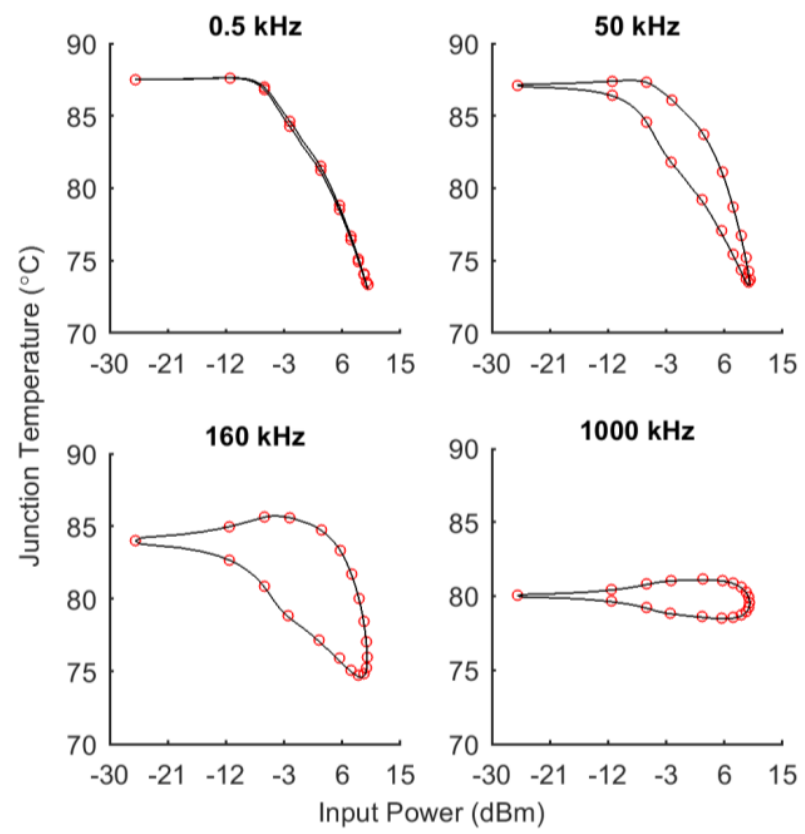

Fig. 6. Junction temperature versus RF power of AM-modulated tone as a function of modulation frequency. DTXM (symbols) and compact electro-thermal model (solid lines). $R_{\mathrm{th}}=140{ }^{\circ} \mathrm{C} / \mathrm{W} . C_{\mathrm{th}}=7.15 \mathrm{E}-9 \mathrm{~J} /{ }^{\circ} \mathrm{C}$.

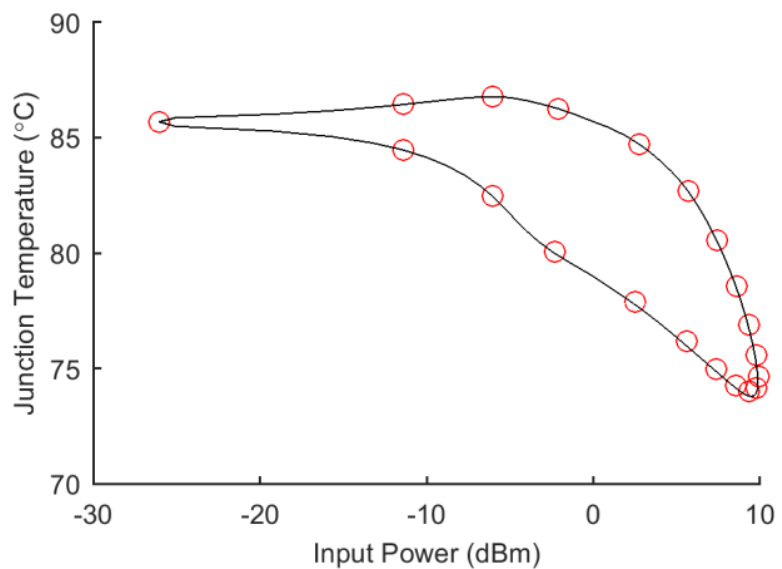

Fig. 5. Dynamic simulation of two-tone response of new DTXM model (symbols) and the compact time-domain self-heating model (solid line). The frequency difference between the two tones was $100 \mathrm{kHz}$. This is a parametric plot of junction temperature versus input power, showing thermal hysteresis.

Interpolated values of the X-parameter measurements over an original grid of ambient temperatures are used as input for this thermal re-referencing. We re-define the X-parameters as functions of $T_{j}$ in addition to the incident power, magnitude and phase of incident waves at the output port, the DC bias conditions, and stimulus frequencies. Since computed $T_{j}$ values will not fall on a grid. The order of the independent variables can be re-arranged, using a Matlab script, such that the $T_{j}$ variables become the inner loop of the data structure, where there is no requirement of a regular structure. The measured Xparameter data must be sampled densely enough for good interpolation over the set of experimentally controlled variables, and to cover a wide enough range of ambient temperatures during this re-referencing post-processing step.

In this work, we identify the model $\mathrm{CW} \mathrm{X}$-parameter NVNA measurements and simulations, over a range of fixed ambient temperatures.

\section{DYNAMIC THERMAL RESULTS}

To demonstrate the applicability of this approach we have extracted a DTXM from a measurement-based dynamic electrothermal circuit model. This enables us to compare the DTXM within a two-tone circuit envelope simulation, and demonstrate the prediction of thermal memory and spectral asymmetry phenomena using the new electrothermal behavioural modelling technique.

The dynamic electrothermal compact model of a GaAs pHEMT [11] was used to generate a conventional static Xparameter model using the Keysight W2305ET X-parameter generator, over a range of ambient temperatures. To evaluate dynamic model performance, a two-tone large-signal excitation was used to stimulate the model in ADS. For a tone-separation of $100 \mathrm{kHz}$, the simulation results are given in Fig. 4 and Fig. 5.

The thermal behaviour demonstrated in these results can be understood by the low-pass filtering effect introduced by the thermal resistance and capacitance. For tone separation much lower than the thermal cut-off frequency $\left(f_{c}=1 / 2 \pi R_{t h} C_{t h}\right)$, the transistor will experience large variation in temperature during the modulation cycle. For separation much greater than 
the thermal cut-off frequency the thermal response is too slow and the hysteresis is minimized. However, at modulation frequencies approaching the thermal cut-off frequency the thermal resistance and capacitance allow temperature transients and the onset of cyclical hysteresis. This is summarized in Fig. 6. For tone separations beyond $10 \mathrm{MHz}$ the junction temperature is essentially constant over a modulation cycle.
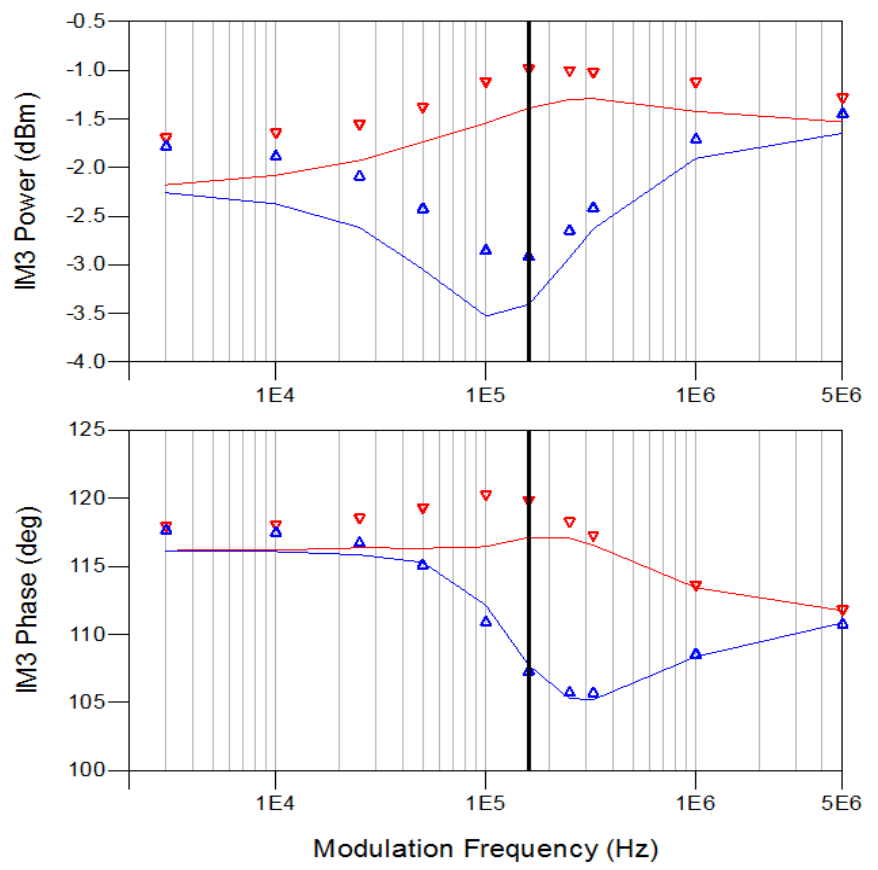

Fig. 7. Magnitude and phase of upper (blue) and lower (red) third order intermodulation products as a function of modulation frequency. DTXM (symbols) and compact model (lines). Thermal cut-off frequency marked as vertical line.

Finally, the intermodulation spectral asymmetry [12] as a function of tone separation is presented in Fig. 7. There is a separation between upper and lower side-bands as the modulation frequency is close to the cut-off frequency defined by the thermal time constant. At lower and higher modulation rates, the magnitudes and phases become much closer. Conventional static X-parameters (not shown) always produce identical magnitudes and phases for the IM3 products at all modulation frequencies. A small difference between the DTXM and compact model are seen in Fig. 7. This difference is due to interpolation errors from the extracted powers and temperatures in the DTXM. This demonstrates that the enhanced DTXM is capable of accurately simulating the important consequences of long-term dynamic thermal memory.

\section{CONCLUSION}

A new dynamic thermal X-parameter model (DTXM) has been presented, and its generation from measurements and simulated data, extraction methodology, and validation demonstrated. The enhanced model predicts the many consequences of dynamic electro-thermal memory phenomena that affects transistors and power amplifiers. This general approach for behavioral modeling leverages the commercially implemented X-parameter measurements on the NVNA, as well as the automated model generator and the built-in static
X-parameter model in ADS, supplementing them with using easy-to-implement auxiliary components. The DTXM makes the behavioral modeling paradigm much more useful and applicable to real-world transistor and power amplifier applications, removing a commonly cited barrier to behavioral modeling adoption for wide-band modulated signal applications.

\section{ACKNOWLEDGMENT}

The authors thank Dr. Jianjun Xu for providing the compact electro-thermal device model from [11]. The authors thank Keysight Technologies for an ACT-UR research grant, and Keysight management for support. This work was also funded and supported by the UK Engineering and Physical Sciences Research Council (EPSRC) grant EP/L02263X/1.

\section{REFERENCES}

[1] P. H. Aaen, J. Plá, and J. Wood, Modeling and Characterization of RF and Microwave Power FETs, Cambridge University Press, 2007.

[2] D. E. Root, "Future device modeling trends," IEEE Microw. Mag., November 2012, pp. 45-59.

[3] P. Roblin, D. E. Root, J. Verspecht, Y. Ko, and J. P. Teyssier, "New trends for the nonlinear measurement and modeling of high-power RF transistors and amplifiers with memory effects," IEEE Trans. Microw. Theory Techn., vol. 60, no. 6, pp. 1964-1978, June, 2012.

[4] D. E. Root, J. Verspecht, J. Horn, and M. Marcu, X-Parameters: Characterization, Modeling, and Design of Nonlinear $R F$ and Microwave Components, Cambridge University Press, 2013.

[5] H. Qi, J. Benedikt and P. J. Tasker, "Data utilization: from direct data lookup to behavioral modeling," IEEE Trans. Microw. Theory Techn., vol. 57, no. 6, pp. 1425-1432, 2009.

[6] "Keysight 2-Port and 4-Port PNA-X network analyzer," Data Sheet and Technical Specifications, Keysight Technologies, 2017. [Online]. https://iterature.cdn.keysight.com/litweb/pdf/N5247-90002.pdf.

[7] J. Verspecht, J. Horn, L. Betts, D. Gunyan, R. Pollard, C. Gillease, and D. E. Root, "Extension of X-parameters to include long-term dynamic memory effects," 2009 IEEE MTT-S Int. Microwave Symp. Dig., June 2009, pp. 741-744

[8] J. Verspecht, J. Horn, and D. E. Root, "A simplified extension of Xparameters to describe memory effects for wideband modulated signals", in Spring 2010 ARFTG Conference proceedings, May 2010.

[9] S. Boumaiza and F. M. Ghannouchi, "Thermal memory effects modeling and compensation in RF power amplifiers and predistortion linearizers," IEEE Trans. Microw. Theory Techn., vol. 51, no. 12, pp. 2427-2433, Dec 2003.

[10] “ATF-54143 low noise enhancement mode pseudomorphic HEMT in a surface mount plastic package," Data Sheet, Broadcom Ltd, 2012. [Online]. https://docs.broadcom.com/docs/AV02-0488EN

[11] J. $\mathrm{Xu}$ and D. E. Root, "Artificial neural networks for compound semiconductor device modeling and characterization," 2017 IEEE CSIC Symposium, Miami, FL, 2017, pp. 1-4.

[12] J. Vuolevi, T. Rahkonen, and J. Manninen, "Measurement technique for characterizing memory effects in RF power amplifiers," IEEE Trans. Microw. Theory Techn., vol. 49, no. 8, pp. 1383-1389, 2001. 